\title{
The eigenvalues of limits of radial Toeplitz operators
}

\author{
Daniel Suárez
}

February 24, 2013

\begin{abstract}
Let $A^{2}$ be the Bergman space on the unit disk. A bounded operator $S$ on $A^{2}$ is called radial if $S z^{n}=\lambda_{n} z^{n}$ for all $n \geq 0$, where $\lambda_{n}$ is a bounded sequence of complex numbers. We characterize the eigenvalues of radial operators that can be approximated by Toeplitz operators with bounded symbols.
\end{abstract}

\section{Introduction and preliminaries}

The Bergman space $A^{2}$ is the closed subspace of analytic functions in $L^{2}(\mathbb{D}, d A)$, where $\mathbb{D}$ is the open unit disk and $d A$ is the normalized area measure. The functions $e_{n}(z):=\sqrt{n+1} z^{n}$, with $n \geq 0$, form the standard orthonormal base of $A^{2}$. We denote by $\mathfrak{L}\left(A^{2}\right)$ the algebra of bounded operators on $A^{2}$. If $a \in L^{\infty}(\mathbb{D})$, the Toeplitz operator with symbol $a$ is

$$
T_{a} f(z):=\int_{D} \frac{a(w) f(w)}{(1-\bar{w} z)^{2}} d A(w), \quad f \in A^{2} .
$$

It is immediate that $\left\|T_{a}\right\| \leq\|a\|_{\infty}$. A Toeplitz operator $T_{a}$ is diagonal with respect to the standard base (i.e.: $T_{a} e_{n}=\lambda_{n} e_{n}$ for some $\lambda_{n} \in \mathbb{C}, n \geq 0$ ) if and only if $a(z)=a(|z|)$ ). By analogy, we say that $S \in \mathfrak{L}\left(A^{2}\right)$ is a radial operator if it is diagonal with respect to the standard base. Radial operators, mostly Toeplitz, have been studied by several authors (see [1], [2], [3] and [7]), mainly because they are among the few Toeplitz operators on $A^{2}$ that we can reasonably understand so far. Despite this fact, some central problems are still open. Consider the space $T_{r a d}:=\left\{T_{b}: b \in L^{\infty}(\mathbb{D})\right.$ radial $\}$, and the Toeplitz algebra

$$
\mathfrak{T}:=\text { the closed subalgebra of } \mathfrak{L}\left(A^{2}\right) \text { generated by }\left\{T_{a}: a \in L^{\infty}(\mathbb{D})\right\} .
$$

In [5] it is proved that any radial operator $S \in \mathfrak{T}$ can be approximated by operators in $T_{\text {rad }}$, whose symbols are constructed from $S$ in a canonical way (see Theorem 4.2 below). Since

\footnotetext{
${ }^{0} 2000$ Mathematics Subject Classification: primary 32A36, secondary 47L80. Key words: radial operators, Toeplitz operators, approximation.
} 
radial operators are determined by their eigenvalues, two problems come readily to mind. Can we characterize the eigenvalue sequences of operators in $T_{\text {rad }}$ ? What about the closure of $T_{\text {rad }}$ ? As we shall see immediately, the first question was settled more than 80 years ago. The present paper deals with the second question.

If $b$ is a bounded radial function, the use of polar coordinates shows that its eigenvalue sequence $\lambda\left(T_{b}\right)$ is given by

$$
\lambda_{n}\left(T_{b}\right)=\left\langle b e_{n}, e_{n}\right\rangle=(n+1) \int_{0}^{1} b(r) r^{2 n} 2 r d r=(n+1) \int_{0}^{1} b\left(t^{1 / 2}\right) t^{n} d t .
$$

That is, a sequence $\left\{\lambda_{n}\right\}$ forms the eigenvalues of a Toeplitz operator with bounded radial symbol if and only if $\left\{\lambda_{n} /(n+1)\right\}$ is the moment sequence of a bounded function on $[0,1]$. In 1921 Hausdorff characterized the moment sequences of measures of bounded variation on the interval $[0,1]$.

Definition 1.1. Let $m \geq 0$ be an integer and $x=\left\{x_{n}\right\}_{n \geq 0}$ be a sequence of complex numbers. The $m$-difference of $x$, denoted $\Delta^{m}(x)$, is the sequence defined by

$$
\Delta_{n}^{m} x:=(-1)^{m} \sum_{j=0}^{m}\left(\begin{array}{c}
m \\
j
\end{array}\right)(-1)^{j} x_{n+j}, \text { for } n \geq 0,
$$

where $\left(\begin{array}{c}m \\ j\end{array}\right)=m ! /(m-j) ! j !$

Further elaboration of Hausdorff's moment theorem showed that given a sequence $x$, there exists a function $a \in L^{\infty}[0,1]$ such that $\int_{0}^{1} a(t) t^{n} d t=x_{n}$ for all $n \geq 0$ if and only if the expression $(k+1)\left(\begin{array}{c}k \\ m\end{array}\right)\left|\Delta_{k-m}^{m} x\right|$ is bounded for all $0 \leq m \leq k$ (see [6, Ch. III]). Together with (1.1), this implies that a sequence $\lambda$ is formed by the eigenvalues of some $T_{b}$, with $b \in L^{\infty}(\mathbb{D})$ radial, if and only if there is a constant $C>0$ such that

$$
(k+1)\left(\begin{array}{c}
k \\
m
\end{array}\right)\left|\Delta_{k-m}^{m} \mu\right| \leq C \text { for all } 0 \leq m \leq k, \text { where } \mu_{n}:=\frac{\lambda_{n}}{n+1} .
$$

Since $\|S\|=\|\lambda(S)\|_{\ell^{\infty}}$ for any radial operator with eigenvalue sequence $\lambda(S)$, it is clear that $S$ is in the closure of $T_{r a d}$ if and only if $\lambda(S)$ is in the $\ell^{\infty}$-closure of the sequences that satisfy (1.2). The obvious inconvenient with this characterization is that this property is very hard to check. Also, it is difficult to construct such sequences without the a priori knowledge that the corresponding operator is a limit of Toeplitz operators with bounded symbols. We provide here two characterizations of these sequences that turn out to be much simpler than (1.2). The resulting eigenvalues consist of the $\ell^{\infty}$-closure of sequences $\lambda$ satisfying any of the conditions:

$$
\sup _{n \geq 0}(n+1)\left|\Delta_{n}^{1} \lambda\right|<\infty \quad \text { or } \quad \sup _{n \geq 0}(n+1)^{2}\left|\Delta_{n}^{2} \lambda\right|<\infty .
$$

This answers affirmatively a question that I posed in [5]. The precise relation between the above conditions and (1.2) is made explicit in Section 4 . 


\subsection{The $n$-Berezin transform}

If $n$ is a nonnegative integer and $z \in \mathbb{D}$, consider the function

$$
K_{z}^{n}(\omega)=\frac{1}{(1-\bar{z} \omega)^{2+n}}(\omega \in \mathbb{D})
$$

When $n=0$ this function is the reproducing kernel for the space $A^{2}$.

Definition 1.2. The $n$-Berezin transform of an operator $S \in \mathfrak{L}\left(A^{2}\right)$ is defined as

$$
B_{n}(S)(z):=(n+1)\left(1-|z|^{2}\right)^{2+n} \sum_{j=0}^{n}\left(\begin{array}{l}
n \\
j
\end{array}\right)(-1)^{j}\left\langle S\left(\omega^{j} K_{z}^{n}\right), \omega^{j} K_{z}^{n}\right\rangle,
$$

where $\langle$,$\rangle is the usual integral pairing.$

It is not difficult to prove that $B_{n}(S) \in L^{\infty}(\mathbb{D}) \cap C^{\infty}(\mathbb{D})$, and that there is a constant $C(n)>0$ such that $\left\|B_{n}(S)\right\|_{\infty} \leq C(n)\|S\|$. If $a \in L^{\infty}(\mathbb{D})$, the binomial expansion of $\left(1-|\omega|^{2}\right)^{n}$ and a conformal change of variables yields

$$
B_{n}(a)(z):=B_{n}\left(T_{a}\right)(z)=\int_{D} a\left(\varphi_{z}(\xi)\right)(n+1)\left(1-|\xi|^{2}\right)^{n} d A(\xi)
$$

where $\varphi_{z}(w)=(z-w) /(1-\bar{z} w)$ is the automorphism of the disk that interchanges 0 and $z$. That is, the above formula defines the $n$-Berezin transform of a function $a \in L^{\infty}(\mathbb{D})$. Since $(n+1)\left(1-|\xi|^{2}\right)^{n} d A(\xi)$ is a probability measure with total mass accumulating at 0 when $n \rightarrow \infty$, it is clear that $B_{n}(a) \rightarrow a$ pointwise if $a \in L^{\infty}(\mathbb{D})$ is continuous. In particular,

$$
B_{n}\left(B_{0}(S)\right) \rightarrow B_{0}(S) \text { for any } S \in \mathfrak{L}\left(A^{2}\right) .
$$

The (conformally) invariant Laplacian is $\tilde{\Delta}=\left(1-|z|^{2}\right)^{2} \Delta$, where $\Delta=\partial \bar{\partial}$ is a quarter of the standard Laplacian, and $\partial, \bar{\partial}$ are the traditional Cauchy-Riemann operators. It is easy to check that $(\tilde{\Delta} f) \circ \psi=\tilde{\Delta}(f \circ \psi)$ for every $f \in C^{2}(\mathbb{D})$ and $\psi \in \operatorname{Aut}(\mathbb{D})$.

We summarize next some of the properties of $B_{n}$ that will be used in the paper. The proofs are in [4]. Let $S \in \mathfrak{L}\left(A^{2}\right)$ and $n \geq 0$. Then

$$
\begin{gathered}
\tilde{\Delta} B_{n}(S)=(n+1)(n+2)\left(B_{n}(S)-B_{n+1}(S)\right) . \\
\left(B_{k} B_{j}\right)(S)=\left(B_{j} B_{k}\right)(S) \text { for all } j, k \geq 0 .
\end{gathered}
$$

Observe that (1.4) implies that $\tilde{\Delta} B_{n}(S) \in L^{\infty}(\mathbb{D})$ for any $S \in \mathfrak{L}\left(A^{2}\right)$, which allows us to further apply $B_{k}$ to this function for any $k \geq 0$. It follows immediately from (1.4) and (1.5) that

$$
\tilde{\Delta} B_{k}\left(B_{n} S\right)=B_{k} \tilde{\Delta}\left(B_{n} S\right) .
$$

Finally, it is easy to prove that if $S \in \mathfrak{L}\left(A^{2}\right)$ is radial, so is the function $B_{n}(S)$. 


\section{Two sequence spaces}

Let $\ell^{\infty}$ be the Banach space of bounded complex sequences indexed from $n \geq 0$.

Definition 2.1. Consider the linear subspaces of $\ell^{\infty}$ :

$$
\begin{aligned}
& d_{1}:=\left\{x \in \ell^{\infty}:\|x\|_{d_{1}}=\sup _{n}(n+1)\left|\Delta_{n}^{1}(x)\right|<\infty\right\} \text { and } \\
& d_{2}:=\left\{x \in \ell^{\infty}:\|x\|_{d_{2}}=\sup _{n}(n+2)^{2}\left|\Delta_{n}^{2}(x)\right|<\infty\right\} .
\end{aligned}
$$

Observe that \|\|$_{d_{1}}$ and \|\|$_{d_{2}}$ are semi-norms that vanish only at constant sequences.

Lemma 2.2. Given $C>4$, for every $n \geq C$ there exists $r \in[1,4]$ such that $m:=\frac{C}{C-r} n$ is the unique integer that satisfies

$$
\sum_{k=n+1}^{m} \frac{C}{k^{2}} \leq \frac{1}{n} \quad \text { and } \quad \sum_{k=n+1}^{m+1} \frac{C}{k^{2}}>\frac{1}{n}
$$

Proof. Fix $C>4$ and suppose that $n \geq C$. For $m \geq n+1$, we have

$$
\frac{1}{2}\left(\frac{1}{n}-\frac{1}{m}\right) \leq \frac{1}{n+1}-\frac{1}{m+1}=\int_{n+1}^{m+1} \frac{1}{x^{2}} d x \leq \sum_{k=n+1}^{m} \frac{1}{k^{2}} \leq \int_{n}^{m} \frac{1}{x^{2}} d x=\frac{1}{n}-\frac{1}{m} .
$$

Straightforward estimates from these inequalities show that

$$
C \sum_{k=n+1}^{m} \frac{1}{k^{2}} \leq \frac{1}{n} \quad \text { if } \quad n+1 \leq m \leq \frac{C}{C-1} n,
$$

and

$$
C \sum_{k=n+1}^{m} \frac{1}{k^{2}} \geq \frac{2}{n} \quad \text { if } \quad m \geq \frac{C}{C-4} n .
$$

Hence, there is $m \in \mathbb{N}$ between $\frac{C}{C-1} n$ and $\frac{C}{C-4} n$ satisfying (2.1). Since the function $f(x)=$ $\frac{C}{C-x} n$ is continuous on $[1,4]$, the mean value theorem gives $r \in[1,4]$ such that $m=\frac{C}{C-r} n$.

Lemma 2.3. Given $\varepsilon>0$, there exists $C=C(\varepsilon)>4$ large enough so that for all $n \geq C$,

$$
E(C, n):=\left[\frac{1}{n}-\frac{C}{(n+1)^{2}}\right]+\ldots+\left[\frac{1}{n}-\sum_{k=n+1}^{m} \frac{C}{k^{2}}\right]<\varepsilon
$$

and

$$
\sum_{k=n+1}^{m} \frac{1}{k}<\varepsilon
$$

where $m=m(C, n)$ is the integer satisfying (2.1). 
Proof. First observe that

$$
E(C, n)=\frac{m-n}{n}-C \sum_{k=n+1}^{m} \frac{(m-k+1)}{k^{2}} \leq \frac{m-n}{n} .
$$

Also, since $\log (1+x) \leq x$ for $x \geq 0$,

$$
\sum_{k=n+1}^{m} \frac{1}{k} \leq \int_{n}^{m} \frac{1}{x} d x=\log \left(\frac{m}{n}\right) \leq \frac{m}{n}-1
$$

By Lemma 2.2 there is $r=r(C, n) \in[1,4]$ such that $m=\frac{C}{C-r} n$. Hence, replacing $m$ by this expression we get

$$
\frac{m}{n}-1=\frac{C}{C-r}-1 \leq \frac{C}{C-4}-1=\frac{4}{C-4}<\varepsilon
$$

if $C$ is large enough.

I learnt the proof of the next proposition from Jorge Antezana (personal communication), to whom I am grateful.

Proposition 2.4. The following statements hold

(1) If $x \in d_{2}$ then $\|x\|_{d_{1}} \leq\|x\|_{d_{2}}$.

(2) ${\overline{d_{1}}}^{\ell^{\infty}}={\overline{d_{2}}}^{\ell^{\infty}}$.

Proof. Let $x \in d_{2}$. Fix $j \geq 0$ and let $n \geq j$. Then

$$
\begin{aligned}
\left|\Delta_{n+1}^{1}(x)-\Delta_{j}^{1}(x)\right| & \leq \sum_{k=j}^{n}\left|\Delta_{k+1}^{1}(x)-\Delta_{k}^{1}(x)\right|=\sum_{k=j}^{n}\left|\Delta_{k}^{2}(x)\right| \\
& \leq \sum_{k=j}^{n} \frac{\|x\|_{d_{2}}}{(k+1)(k+2)}=\|x\|_{d_{2}}\left(\frac{1}{j+1}-\frac{1}{n+2}\right) .
\end{aligned}
$$

Hence, $\Delta_{n+1}^{1}(x)$ is a Cauchy sequence, and since the sequence $x$ is bounded, $\Delta_{n+1}^{1}(x) \rightarrow 0$. Taking limit when $n \rightarrow \infty$ in (2.4) we obtain $\left|\Delta_{j}^{1}(x)\right| \leq \frac{\|x\|_{d_{2}}}{j+1}$ for $j \geq 0$. This proves (1). In particular, $d_{2} \subseteq d_{1}$, and the proof of (2) is reduced to see that $d_{1} \subset{\overline{d_{2}}}^{\infty}$. So, let $x \in d_{1}$ and $\varepsilon>0$. We can assume without loss of generality that $\|x\|_{d_{1}} \leq 1$ and that $x_{n} \in \mathbb{R}$ for every $n \geq 0$. Pick $C=C(\varepsilon)>4$ as in Lemma 2.3 and define $y \in d_{2}$ as:

$$
y_{n}= \begin{cases}x_{n} & \text { if } n \leq \max \{2 / \varepsilon, C\} \\ y_{n-1}+\delta & \text { if } n>\max \{2 / \varepsilon, C\}\end{cases}
$$


where $\delta$ minimizes $\left|\left(y_{n-1}+\delta\right)-x_{n}\right|$ under the restrictions $|\delta| \leq \frac{1}{n},\left|\left(y_{n-1}-y_{n-2}\right)-\delta\right| \leq \frac{C}{n^{2}}$.

We aim to prove that $\|x-y\|_{\infty} \leq 5 \varepsilon$. First observe that given $n_{0}$ such that $\left|x_{n_{0}}-y_{n_{0}}\right|<\varepsilon$, it is enough to estimate $\left|x_{n}-y_{n}\right|$ for all the subsequent values of $n$ until the first time that $\operatorname{sgn}\left(x_{n}-y_{n}\right) \neq \operatorname{sgn}\left(x_{n+1}-y_{n+1}\right)$, because this change of sign implies that $\left|x_{n+1}-y_{n+1}\right| \leq$ $2 /(n+1)<\varepsilon$. So, suppose that $n_{0}$ is such that $x_{n_{0}}<y_{n_{0}}$ (the analysis is symmetrical for $\left.x_{n_{0}}>y_{n_{0}}\right)$, and let $n_{1}$ be the first integer $>n_{0}$ such that $x_{n_{1}} \geq y_{n_{1}}$. We estimate how much $y_{n}-x_{n}$ can grow for $n_{0}<n<n_{1}$. Since $x_{n}<y_{n}$ for those values of $n$,

$$
y_{n}-y_{n-1}=y_{n-1}-y_{n-2}-\frac{C}{n^{2}}=\left(y_{n_{0}}-y_{n_{0}-1}\right)-\sum_{k=n_{0}+1}^{n} \frac{C}{k^{2}}
$$

for all $n_{0}<n<n_{1}$. Consider two cases.

Case 1: $y_{n_{0}}-y_{n_{0}-1} \leq 0$. It follows from (2.5) that for $n_{0}<n<n_{1}$,

$$
\begin{aligned}
y_{n}-x_{n} & =\left(y_{n_{0}}-x_{n_{0}}\right)+\left(y_{n}-y_{n_{0}}\right)-\left(x_{n}-x_{n_{0}}\right) \\
& \leq\left(y_{n_{0}}-x_{n_{0}}\right)-\left[\frac{C}{\left(n_{0}+1\right)^{2}}+\cdots+\sum_{k=n_{0}+1}^{n} \frac{C}{k^{2}}\right]-\sum_{j=n_{0}+1}^{n}\left(x_{j}-x_{j-1}\right) \\
& \leq\left(y_{n_{0}}-x_{n_{0}}\right)+\left[\left|x_{n_{0}+1}-x_{n_{0}}\right|-\frac{C}{\left(n_{0}+1\right)^{2}}\right]+\cdots+\left[\left|x_{n}-x_{n-1}\right|-\sum_{k=n_{0}+1}^{n} \frac{C}{k^{2}}\right]
\end{aligned}
$$

Let $m_{0}>n_{0}$ be the integer associated with $n_{0}$ by (2.1). If $n>m_{0}$, (2.1) and $\left|x_{n}-x_{n-1}\right| \leq$ $\frac{1}{n} \leq \frac{1}{n_{0}}$ imply that the corresponding summand in square brackets must be negative. Thus,

$$
\begin{aligned}
y_{n}-x_{n} & \leq\left(y_{n_{0}}-x_{n_{0}}\right)+\left[\frac{1}{n_{0}}-\frac{C}{\left(n_{0}+1\right)^{2}}\right]+\cdots+\left[\frac{1}{n_{0}}-\sum_{k=n_{0}+1}^{m_{0}} \frac{C}{k^{2}}\right] \\
& =\left(y_{n_{0}}-x_{n_{0}}\right)+E\left(C, n_{0}\right) \leq\left(y_{n_{0}}-x_{n_{0}}\right)+\varepsilon
\end{aligned}
$$

where the las inequality comes from (2.2).

Case 2: $y_{n_{0}}-y_{n_{0}-1}>0$. If $n_{0} \leq n<n_{1}$ is any integer such that

$$
y_{k}-y_{k-1}>0 \text { for } k=n_{0}, \ldots, n \text {, }
$$

then (2.5) implies that

$$
0<y_{n}-y_{n-1}=\left(y_{n_{0}}-y_{n_{0}-1}\right)-\sum_{k=n_{0}+1}^{n} \frac{C}{k^{2}} \leq \frac{1}{n_{0}}-\sum_{k=n_{0}+1}^{n} \frac{C}{k^{2}},
$$


which together with the definition of $m_{0}$ forces $n \leq m_{0}$. So, (2.2) and (2.3) give

$$
\begin{aligned}
y_{n}-x_{n} & =\left(y_{n_{0}}-x_{n_{0}}\right)+\left(y_{n}-y_{n_{0}}\right)-\left(x_{n}-x_{n_{0}}\right) \\
& \leq\left(y_{n_{0}}-x_{n_{0}}\right)+\left[\frac{1}{n_{0}}-\frac{C}{\left(n_{0}+1\right)^{2}}\right]+\cdots+\left[\frac{1}{n_{0}}-\sum_{k=n_{0}+1}^{m_{0}} \frac{C}{k^{2}}\right]+\sum_{k=n_{0}+1}^{m_{0}}\left|x_{k}-x_{k-1}\right| \\
& \leq\left(y_{n_{0}}-x_{n_{0}}\right)+E\left(C, n_{0}\right)+\sum_{k=n_{0}+1}^{m_{0}} \frac{1}{k} \leq\left(y_{n_{0}}-x_{n_{0}}\right)+2 \varepsilon
\end{aligned}
$$

If $n$ is the largest integer satisfying (2.6), then either $n+1=n_{1}$ (and we are done) or $n+1$ is in Case 1, meaning that $y_{n+1}-y_{n} \leq 0$ (while $y_{n+1}>x_{n+1}$ ). Hence, the estimate of Case 1 and (2.7) show that for all $n+1 \leq k<n_{1}$,

$$
y_{k}-x_{k} \leq\left(y_{n+1}-x_{n+1}\right)+\varepsilon \leq \frac{2}{(n+1)}+\left(y_{n}-x_{n}\right)+\varepsilon<\varepsilon+\left(y_{n_{0}}-x_{n_{0}}\right)+2 \varepsilon+\varepsilon .
$$

That is, we have shown that $y_{k}-x_{k} \leq y_{n_{0}}-x_{n_{0}}+4 \varepsilon$ for all $n_{0} \leq k<n_{1}$. By the symmetry of the case $x_{n_{0}}>y_{n_{0}}$ and the comments that follow the definition of $y$, we get $\|y-x\|_{\infty}<5 \varepsilon$.

\section{The invariant Laplacian of an operator}

\section{Definition 3.1. Let}

$$
\mathfrak{D}=\left\{S \in \mathfrak{L}\left(A^{2}\right): \exists T \in \mathfrak{L}\left(A^{2}\right) \text { such that } \tilde{\Delta} B_{0}(S)=B_{0}(T)\right\}
$$

and define $\tilde{\Delta}: \mathfrak{D} \rightarrow \mathfrak{L}\left(A^{2}\right)$ by $\tilde{\Delta} S=T$.

Lemma 3.2. If $S_{n}, S \in \mathfrak{L}\left(A^{2}\right)$, with $S_{n} \rightarrow S$ in the weak operator topology. Then

$$
B_{0}\left(S_{n}\right) \rightarrow B_{0}(S) \text { and } \tilde{\Delta} B_{0}\left(S_{n}\right) \rightarrow \tilde{\Delta} B_{0}(S) \text { pointwise. }
$$

Proof. We only prove the assertion for $\tilde{\Delta} B_{0}$, since the proofs are analogous. It is clear that if $k$ is a non negative integer, $\bar{\partial}_{z}\left(\bar{z}^{k} K_{z}\right)(w)$ is a bounded analytic function of $w$. Thus,

$$
\Delta|z|^{2 k}\left\langle S_{n} K_{z}, K_{z}\right\rangle=\left\langle S_{n} \bar{\partial}_{z}\left(\bar{z}^{k} K_{z}\right), \bar{\partial}_{z}\left(\bar{z}^{k} K_{z}\right)\right\rangle \rightarrow\left\langle S \bar{\partial}_{z}\left(\bar{z}^{k} K_{z}\right), \bar{\partial}_{z}\left(\bar{z}^{k} K_{z}\right)\right\rangle=\Delta|z|^{2 k}\left\langle S K_{z}, K_{z}\right\rangle,
$$

with point convergence on $z$. In particular,

$$
\tilde{\Delta} B_{0}\left(S_{n}\right)=\left(1-|z|^{2}\right)^{2} \Delta\left[\left(1+|z|^{4}-2|z|^{2}\right)\left\langle S_{n} K_{z}, K_{z}\right\rangle\right]
$$

converges pointwise to $\tilde{\Delta} B_{0}(S)$. 
Lemma 3.3. For $\lambda \in d_{2}$ consider the sequence $\gamma$ given by

$$
\gamma_{n}:= \begin{cases}2\left(\lambda_{1}-\lambda_{0}\right), & \text { if } n=0 \\ (n+1)\left[(n+2)\left(\lambda_{n+1}-\lambda_{n}\right)-n\left(\lambda_{n}-\lambda_{n-1}\right)\right], & \text { if } n \geq 1\end{cases}
$$

Then

$$
6^{-1}\|\lambda\|_{d_{2}} \leq\|\gamma\|_{\infty} \leq 6\|\lambda\|_{d_{2}} .
$$

Proof. Setting $\lambda_{-1}=0$, for $n \geq 0$ we have

$$
\begin{aligned}
\gamma_{n} & =(n+2)(n+1) \Delta_{n}^{1}(\lambda)-(n+1) n \Delta_{n-1}^{1}(\lambda) \\
& =(n+2) b_{n+1}-(n+1) b_{n},
\end{aligned}
$$

where $b_{n}:=n \Delta_{n-1}^{1}(\lambda)$. Therefore

$$
(n+2)\left|b_{n+1}\right|=\left|(n+2) b_{n+1}-1 b_{0}\right|=\left|\sum_{j=0}^{n}\left[(j+2) b_{j+1}-(j+1) b_{j}\right]\right| \leq(n+1)\|\gamma\|_{\infty},
$$

leading to $(n+1)\left|\Delta_{n}^{1}(\lambda)\right|=\left|b_{n+1}\right| \leq\|\gamma\|_{\infty}$, for $n \geq 0$. That is, $\|\lambda\|_{d_{1}} \leq\|\gamma\|_{\infty}$. On the other hand, if $n \geq 1$,

$$
\begin{aligned}
\gamma_{n} & =(n+1)\left[(n+2)\left(\lambda_{n+1}-2 \lambda_{n}+\lambda_{n-1}\right)+2\left(\lambda_{n}-\lambda_{n-1}\right)\right] \\
& =\left(\frac{n+2}{n+1}\right)(n+1)^{2} \Delta_{n-1}^{2}(\lambda)+2\left(\frac{n+1}{n}\right) n \Delta_{n-1}^{1}(\lambda) .
\end{aligned}
$$

Hence,

$$
\|\gamma\|_{\ell^{\infty}} \leq 2\|\lambda\|_{d_{2}}+4\|\lambda\|_{d_{1}} \stackrel{\text { by Prop. } 2.4}{\leq} 6\|\lambda\|_{d_{2}}
$$

and since

$$
\left|(n+1)^{2} \Delta_{n-1}^{2}(\lambda)\right|=\left|\frac{(n+1)}{(n+2)} \gamma_{n}-2 \frac{(n+1)^{2}}{(n+2) n} n \Delta_{n-1}^{1}(\lambda)\right| \leq\left|\gamma_{n}\right|+4 n\left|\Delta_{n-1}^{1}(\lambda)\right|,
$$

then

$$
\|\lambda\|_{d_{2}} \leq\|\gamma\|_{\ell^{\infty}}+4\|\lambda\|_{d_{1}} \leq 5\|\gamma\|_{\ell^{\infty}}
$$

The orthogonal projection onto the subspace generated by $e_{n}$ is $E_{n} f=\left\langle f, e_{n}\right\rangle e_{n}$, where $n \geq 0$ and $f \in A^{2}$. Thus, a bounded operator $S$ is radial if and only if it can be written as $S=\sum_{n \geq 0} \lambda_{n} E_{n}$, where $\lambda \in \ell^{\infty}$ is the sequence of its eigenvalues. Also, observe that the reproducing property of $K_{z}^{0}$ shows that

$$
\left(1-|z|^{2}\right)^{2} B_{0}\left(E_{n}\right)(z)=\left(1-|z|^{2}\right)^{2}\left\langle K_{z}^{0}, e_{n}\right\rangle\left\langle e_{n}, K_{z}^{0}\right\rangle=\left(1-|z|^{2}\right)^{2}\left|e_{n}(z)\right|^{2} .
$$


Proposition 3.4. Let $S \in \mathfrak{L}\left(A^{2}\right)$ be a radial operator with eigenvalue sequence $\lambda$. Then $S \in \mathfrak{D}$ if and only if $\lambda \in d_{2}$, in which case

$$
\tilde{\Delta} \sum_{n \geq 0} \lambda_{n} E_{n}=\sum_{n \geq 0} \gamma_{n} E_{n}
$$

where $\gamma$ is given by (3.1). Thus, $6^{-1}\|\lambda\|_{d_{2}} \leq\|\tilde{\Delta} S\| \leq 6\|\lambda\|_{d_{2}}$.

Proof. Since the partial sums of $\sum \lambda_{n} E_{n}$ tend to $S$ in the strong operator topology, Lemma 3.2 implies that

$$
\tilde{\Delta} B_{0}\left(\sum \lambda_{n} E_{n}\right)=\sum \lambda_{n} \tilde{\Delta} B_{0}\left(E_{n}\right)
$$

By (3.2),

$$
\begin{aligned}
\tilde{\Delta} B_{0}\left(E_{n}\right)(z) & =(n+1)\left(1-|z|^{2}\right)^{2} \Delta\left(1-|z|^{2}\right)^{2}|z|^{2 n} \\
& =(n+1)\left(1-|z|^{2}\right)^{2}\left(n^{2}\left|z^{n-1}\right|^{2}+(n+2)^{2}\left|z^{n+1}\right|^{2}-2(n+1)^{2}\left|z^{n}\right|^{2}\right) .
\end{aligned}
$$

Then

$$
\begin{aligned}
\tilde{\Delta} B_{0}(S) & =\left(1-|z|^{2}\right)^{2} \sum_{n \geq 0} \lambda_{n}(n+1)\left(n^{2}|z|^{2(n-1)}+(n+2)^{2}|z|^{2(n+1)}-2(n+1)^{2}|z|^{2 n}\right) \\
& =\left(1-|z|^{2}\right)^{2} \sum_{n \geq 0}|z|^{2 n}(n+1)^{2}\left[(n+2) \lambda_{n+1}+n \lambda_{n-1}-2(n+1) \lambda_{n}\right] \\
& =\left(1-|z|^{2}\right)^{2} \sum_{n \geq 0}\left|e_{n}(z)\right|^{2}(n+1)\left[(n+2) \lambda_{n+1}+n \lambda_{n-1}-2(n+1) \lambda_{n}\right],
\end{aligned}
$$

where we are taking $\lambda_{-1}=0$, and the second equality comes from regrouping the series, which is absolutely and uniformly convergent on compact sets of $\mathbb{D}$. That is,

$$
\tilde{\Delta} B_{0}(S)=\left(1-|z|^{2}\right)^{2} \sum_{n \geq 0} \gamma_{n}\left|e_{n}(z)\right|^{2}
$$

where $\gamma_{n}=(n+1)\left[(n+2) \lambda_{n+1}+n \lambda_{n-1}-2(n+1) \lambda_{n}\right]$. If $\lambda \in d_{2}$, Lemma 3.3 says that $\gamma \in \ell^{\infty}$. So, the operator $T:=\sum_{n \geq 0} \gamma_{n} E_{n}$ is bounded, and (3.2) with Lemma 3.2 imply that

$$
B_{0}(T)=\left(1-|z|^{2}\right)^{2} \sum_{n \geq 0} \gamma_{n}\left|e_{n}(z)\right|^{2}=\tilde{\Delta} B_{0}(S) .
$$

Reciprocally, suppose that $T$ is a bounded operator that satisfies $B_{0}(T)=\tilde{\Delta} B_{0}(S)$. Writing $K_{z}^{0}(w)=\sum \overline{e_{m}(z)} e_{m}(w)$ we get

$$
B_{0}(T)(z)=\left(1-|z|^{2}\right)^{2}\left\langle T K_{z}^{0}, K_{z}^{0}\right\rangle=\left(1-|z|^{2}\right)^{2} \sum_{n, m=0}^{\infty}\left\langle T e_{n}, e_{m}\right\rangle \overline{e_{n}(z)} e_{m}(z)
$$


which clearly implies that $\left\langle T e_{n}, e_{m}\right\rangle=0$ for $n \neq m$ and $\left\langle T e_{n}, e_{n}\right\rangle=\gamma_{n}$. Therefore, $\gamma \in \ell^{\infty}$ and Lemma 3.3 implies that $\lambda \in d_{2}$.

In either case, $\tilde{\Delta} S=\sum \gamma_{n}\left(e_{n} \otimes e_{n}\right)$, which proves $(\underline{3.3})$, and since $\|\tilde{\Delta} S\|=\|\gamma\|_{\ell^{\infty}}$, the last assertion of the proposition follows from Lemma 3.3 ,

\section{Approximation by radial Toeplitz operators}

Lemma 4.1. Suppose that $S \in \mathfrak{L}\left(A^{2}\right)$ is such that $\left\|T_{\tilde{\Delta} B_{k}(S)}\right\| \leq C$ independently of $k$. Then $T_{B_{k}(S)} \rightarrow S$.

Proof. By (1.4), $T_{\tilde{\Delta} B_{k}(S)}=(k+1)(k+2)\left(T_{B_{k}(S)}-T_{B_{k+1}(S)}\right)$. So,

$$
T_{B_{0}(S)}-\sum_{k=0}^{m} \frac{T_{\tilde{\Delta} B_{k}(S)}}{(k+1)(k+2)}=T_{B_{m+1}(S)}
$$

and since $\left\|T_{\tilde{\Delta} B_{k}(S)}\right\| \leq C$, the series of the norms is convergent, which implies the convergence of $T_{B_{m}(S)}$, say to $R \in \mathfrak{L}\left(A^{2}\right)$. Since $B_{0}$ is a bounded operator from $\mathfrak{L}\left(A^{2}\right)$ in $L^{\infty}$, we also have that $B_{0}\left(T_{B_{m}(S)}\right) \rightarrow B_{0}(R)$ in $L^{\infty}$-norm. On the other hand, (1.5) and (1.3) imply

$$
B_{0}\left(T_{B_{m}(S)}\right)=B_{0} B_{m}(S)=B_{m} B_{0}(S) \rightarrow B_{0}(S) \text { pointwise. }
$$

This means that $B_{0}(S)=B_{0}(R)$, and since $B_{0}$ is one-to-one, $S=R$.

We recall that the Toeplitz algebra, $\mathfrak{T}$, is formed by all the operators that can be approximated by polynomials of Toeplitz operators with bounded symbols. The two results in the following theorem are Corollary 3.2 and Theorem 3.3 of [5], respectively.

Theorem 4.2. Let $S \in \mathfrak{L}\left(A^{2}\right)$ be a radial operator. Then

1. $\left\|T_{B_{k}(S)}\right\| \leq\|S\|$.

2. $S \in \mathfrak{T}$ if and only if $T_{B_{k}(S)} \rightarrow S$.

It is easy now to finish the proof of the main result in this paper.

Theorem 4.3. Let $S \in \mathfrak{L}\left(A^{2}\right)$ be a radial operator with eigenvalue sequence $\lambda(S)$. Then the following statements are equivalent

(1) $S \in \mathfrak{T}$ (or equivalently, $T_{B_{k}(S)} \rightarrow S$ )

(2) $\lambda(S) \in \bar{d}_{2}^{\ell^{\infty}}$

(3) $\lambda(S) \in \bar{d}_{1}^{\ell^{\infty}}$ 
Proof. Observe that Proposition 2.4 gives the equivalence between (2) and (3). We shall prove that (1) is equivalent to (2). It is quite easy to show that if $b$ is a bounded radial function then its eigenvalue sequence $\lambda\left(T_{b}\right)$ is in $d_{2}$. Indeed, if $n \geq 1$, (1.1) yields

$$
\left|\Delta_{n-1}^{2}\left(\lambda\left(T_{b}\right)\right)\right| \leq \int_{0}^{1}\left|b\left(t^{1 / 2}\right)\right|\left|(n+2) t^{n+1}-2(n+1) t^{n}+n t^{n-1}\right| d t \leq \frac{8\|b\|_{\infty}}{(n+2)^{2}} .
$$

If (1) holds then

$$
\lambda\left(T_{B_{k}(S)}\right) \stackrel{\ell^{\infty}}{\rightarrow} \lambda(S) \quad \text { when } \quad n \rightarrow \infty .
$$

So, $\lambda(S) \in \bar{d}_{2}^{\ell^{\infty}}$. Now suppose that $\lambda(S)$ is the $\ell^{\infty}$-limit of a sequence $\lambda_{j}$ contained in $d_{2}$ (here $\lambda_{j}$ denotes the whole sequence, not the $j$-entry of a sequence). If $S_{j}$ is the radial operator with eigenvalues $\lambda\left(S_{j}\right)=\lambda_{j}$, then $S_{j} \rightarrow S$ in $\mathfrak{L}\left(A^{2}\right)$-norm. If we show that $T_{B_{k}\left(S_{j}\right)} \rightarrow S_{j}$ when $k \rightarrow \infty$ for every fixed value of $j$, then $S \in \mathfrak{T}$ and (1) will follow. That is, we can assume that $\lambda(S) \in d_{2}$. By Proposition 3.4 then $S \in \mathfrak{D}$ and $\|\tilde{\Delta} S\| \leq 6\|\lambda(S)\|_{d_{2}}$. Since $\tilde{\Delta} S$ is a radial operator, Theorem 4.2 says that $\left\|T_{B_{k}(\tilde{\Delta} S)}\right\| \leq\|\tilde{\Delta} S\|$. Furthermore, by (1.5) and (1.6),

$$
B_{0} \tilde{\Delta} B_{k}(S)=\tilde{\Delta} B_{0} B_{k}(S)=\tilde{\Delta} B_{k} B_{0}(S)=B_{k} \tilde{\Delta} B_{0}(S)=B_{k} B_{0}(\tilde{\Delta} S)=B_{0} B_{k}(\tilde{\Delta} S)
$$

and since $B_{0}$ is one-to-one, $\tilde{\Delta} B_{k}(S)=B_{k}(\tilde{\Delta} S)$. Putting all this together gives

$$
\left\|T_{\tilde{\Delta} B_{k}(S)}\right\|=\left\|T_{B_{k}(\tilde{\Delta} S)}\right\| \leq\|\tilde{\Delta} S\| \leq 6\|\lambda(S)\|_{d_{2}}
$$

for all $k$. Lemma 4.1 then says that $T_{B_{k}(S)} \rightarrow S$.

A direct comparison between the conditions defining $d_{1}$ and $d_{2}$ with (1.2) shows that a sequence $\lambda$ satisfies (1.2) for

$$
\begin{aligned}
& m=0 \text { and } k \geq 0 \Leftrightarrow \lambda \in \ell^{\infty}, \\
& m=0,1 \text { and } k \geq m \Leftrightarrow \lambda \in d_{1}, \\
& m=0,1,2 \text { and } k \geq m \Leftrightarrow \lambda \in d_{2} .
\end{aligned}
$$

Therefore, if for any integer $p \geq 1$ we define

$$
d_{p}:=\left\{x \in \mathbb{C}^{\mathbb{N}_{0}}: x \text { satisfies (1.2) for } m=0, \ldots, p \text { and } k \geq m\right\},
$$

then $d_{p+1} \subset d_{p} \subset \ell^{\infty}$, and the comment that follows (1.2) together with Theorem 4.3 yield

$$
\bigcap_{p \geq 1} d_{p}{ }^{\infty}=\bar{d}_{1}^{\ell^{\infty}}
$$

In particular, an immediate consequence is the second assertion of Proposition 2.4. However, the assertion should be proved independently of this equality in order to avoid a cyclic argument. 
Next we see two applications of the theorem. Formula (1.1) defines a sequence $\lambda(b)$ for any radial function $b \in L^{1}(\mathbb{D})$, with

$$
\lambda_{n}(b)=(n+1) \int_{0}^{1} b\left(t^{1 / 2}\right) t^{n} d t, \text { for } n \geq 0 .
$$

So, $b$ induces a bounded Toeplitz operator $T_{b}$ on $A^{2}$ if and only if the sequence $\lambda(b)$ is bounded, with $\left\|T_{b}\right\|=\|\lambda(b)\|_{\ell_{\infty}}$. To this writing I do not know any geometric necessary and sufficient condition on $b$ for this to hold. However, there is a well-known sufficient condition:

$$
\left|\int_{t}^{1} b\left(x^{1 / 2}\right) d x\right| \leq C(1-t) \text { for all } t \in[0,1]
$$

which turns out to be necessary when $b \geq 0$. Actually, when $b \geq 0$, (4.1) is a particular case of a more general situation involving Carleson measures for Bergman spaces. The next corollary shows that if $b$ satisfies (4.1) then $T_{b}$ is not only bounded, but it belongs to the Toeplitz algebra $\mathfrak{T}$, and even to $\mathfrak{D}$.

Corollary 4.4. Let $b \in L^{1}(\mathbb{D})$ be a radial function satisfying (4.1). Then $\|\lambda(b)\|_{\ell^{\infty}} \leq C$ and $\|\lambda(b)\|_{d_{2}} \leq 10 C$. In particular, $T_{b} \in \mathfrak{D}$ and hence, in $\mathfrak{T}$ (by Prop. 3.4 and Thm. 4.3).

Proof. For $n \geq 1$, integration by parts gives

$$
\lambda_{n}(b)=\int_{0}^{1}\left[\int_{t}^{1} b\left(x^{1 / 2}\right) d x\right](n+1) n t^{n-1} d t .
$$

Using (4.1) we immeditely see that $\left|\lambda_{n}(b)\right| \leq C$ for $n \geq 1$. For $n \geq 2$ :

$$
\begin{aligned}
\left|\Delta_{n-1}^{2}(\lambda(b))\right| & \leq C \int_{0}^{1}(1-t) t^{n-2}\left|(n+2)(n+1) t^{2}-2(n+1) n t+n(n-1)\right| d t \\
& =C \int_{0}^{1}(1-t) t^{n-2}\left|(n+1) n(1-t)^{2}+2 n\left(t^{2}-1\right)+2 t^{2}\right| d t \\
& \leq 2 C \int_{0}^{1}(1-t) t^{n-2}\left[n^{2}(1-t)^{2}+t^{2}\right] d t \\
& =2 C\left[n^{2} \frac{3 !(n-2) !}{(n+2) !}+\frac{n !}{(n+2) !}\right] \leq \frac{10 C}{(n+1)^{2}},
\end{aligned}
$$

where the last equality comes from $\int_{0}^{1}(1-t)^{p} t^{q} d t=p ! q ! /(p+q+1)$ ! for integers $p, q \geq 0$. Since $\left|\lambda_{0}(b)\right|=\left|\int_{0}^{1} b\left(x^{1 / 2}\right) d x\right| \leq C$ by (4.1), and $\left|\Delta_{0}^{2}(\lambda)\right| \leq\left|\lambda_{2}\right|+2\left|\lambda_{1}\right|+\left|\lambda_{0}\right| \leq 3 C$, the corollary follows.

It is known that if $S \in \mathfrak{L}\left(A^{2}\right)$ is diagonal, then its essential spectrum $\sigma_{e}(S)$ is formed by the limit points of its eigenvalues. In particular, since $\Delta_{n}^{1}(\lambda(b)) \rightarrow 0$ for any radial $b \in L^{1}(\mathbb{D})$, 
then $\sigma_{e}\left(T_{b}\right)$ is connected whenever $T_{b}$ is bounded. Since also $\Delta_{n}^{1}(\lambda) \rightarrow 0$ when $\lambda$ belongs to the $\ell^{\infty}$-closure of $d_{1}$, Theorem 4.3 implies that $\sigma_{e}(S)$ is connected for every radial $S \in \mathfrak{T}$. In [2, Coro. 2.10], Grudsky and Vasilevski show examples of compact sets that can be the essential spectrum of $T_{b}$, for $b \in L^{\infty}(\mathbb{D})$ radial. We finish this paper by showing that if instead of $T_{\text {rad }}$ we take its closure, any nonempty, compact, connected set is the essential spectrum of some operator in this class.

Corollary 4.5. Let $E \subset \mathbb{C}$ be a nonempty, compact, connected set. Then there is a radial operator $S \in \mathfrak{T}$ such that $\sigma_{e}(S)=E$.

Proof. It is easy to construct a sequence $\lambda \in d_{1}$ whose limit points are exactly the points of $E$. If $S$ is the radial operator with eigenvalue sequence $\lambda$, then $\sigma_{e}(S)=E$, and Theorem 4.3 says that $S \in \mathfrak{T}$.

Acknowledgements: The author is partially supported by the Ramón y Cajal program and the grants MTM2005-00544 and 2005SGR00774, from the State Secretary of Education and Universities, Spain.

\section{References}

[1] P. Ahern and Z. Čučković, Some examples related to the Brown-Halmos Theorem for the Bergman space, Acta Sci. Math. 70, No.1-2 (2004), 373-378.

[2] S. Grudsky and N. Vasilevski, Bergman-Toeplitz operators: Radial component influence, Integral Equations Oper. Theory 40, No.1 (2001) 16-33.

[3] B. Korenblum and K. Zhu, An application of Tauberian theorems to Toeplitz operators, Journal. Op. Theory 33 (1995), 353-361.

[4] D. Suárez, Approximation and symbolic calculus for Toeplitz algebras on the Bergman space, Rev. Mat. Iberoamericana 20 (2004), 563-610.

[5] D. Suárez, Approximation and the n-Berezin transform of operators on the Bergman space, J. Reine Angew. Math. 581 (2005), 175-192.

[6] D. V. Widder, 'The Laplace Transform', Princeon Univ. Press, Princeton (1972).

[7] N. Zorboska, The Berezin transform and radial operators. Proc. Amer. Math. Soc. 131, No.3 (2003), 793-800.

Daniel Suárez

Departament de Matemàtiques 
Universitat Autònoma de Barcelona

08193, Bellaterra, Barcelona

Spain

dsuarez@mat.uab.es 